\title{
ORDERINGS FOR NONCOMMUTATIVE RINGS
}

\author{
Thomas C. Craven
}

Introduction and Notation. The goal of this paper is to present the beginnings of a theory of real algebraic geometry for noncommutative rings. For a basic introduction to the commutative theory, see Lam $[\mathbf{L}]$. The word field will be used in this paper to mean a (generally noncommutative) skewfield; we shall specify a commutative field when we need to. $R$ will denote a noncommutative ring with 1. We shall define a concept of ordering for $R$ which we show behaves properly with respect to orderings of "residue fields" and generalizes the usual concepts of orderings for fields and commutative rings. In the final section, we take a brief look at the real spectrum. The complications of this theory can be avoided for special classes of noncommutative rings such as Ore domains (see, for example, $[\mathbf{P}]$ ).

Let $\mathcal{M}(R)$ denote the set of all square matrices over $R$. The notation $(a \mid A)$ will be used to denote an augmented matrix with $a$ as the first column and $A$ as the remainder of the matrix. The basic concepts for noncommutative algebraic geometry which we use are due to P. M. Cohn $[$ Co1, Co2, Co3 $]$ and we shall generally use his notation. In addition to the usual matrix operations, we will use $A+B$ to denote the matrix $\left[\begin{array}{cc}A & 0 \\ 0 & B\end{array}\right]$. If two matrices $A$ and $B$ are identical except for one row (or one column), then $A \nabla B$ denotes the matrix identical to $A$ and $B$ in all entries except that the entries in the special row (column) are added. Note that for commutative rings $R$, we have $\operatorname{det}(A \nabla B)=\operatorname{det} A+\operatorname{det} B$. For this reason, the operation $\nabla$ is referred to as the determinantal sum. Cohn has shown that, in analogy to the residue field of a localization of a commutative ring at a prime ideal, one can study epic R-fields. These are fields generated by the homomorphic image of $R$ inside them. They are determined, not by a prime ideal, but by a "prime matrix ideal" $\wp$. We shall denote the corresponding epic R-field by $K(\wp)$; the matrix ideal $\wp$ is called the singular kernel of the mapping from $R$ to $K(\wp)$.

An $n \times n$ matrix $A$ is called nonfull if $A=B C$, where $B$ is $n \times r, C$ is $r \times n$ and $r<n$. A matrix ideal is a subset $\wp$ of $\mathcal{M}(R)$ which contains all nonfull matrices, is closed under $\dot{+}$ and $\nabla$ when defined, and contains a matrix $A$ whenever it contains $A \dot{+}$. A matrix ideal $\wp$ is called a prime matrix ideal if it is a proper subset of $\mathcal{M}(R)$ and satisfies $A \dot{+} B \in \wp \Longrightarrow A \in \wp$ or $B \in \wp$.

The real theory. We now look at how orderings can be included in the algebraic geometry developed by Cohn. We thank Jim Madden for pointing out that some of these ideas were developed earlier (and independently) by Gábor Révész $[\mathbf{R}]$. His approach and goals are somewhat different than ours, but a maximal matrix cone in his paper can be seen to be equivalent to a matrix ordering as defined below. Our approach will be far more natural for anyone familiar with the commutative 
theory. Also, we provide a proof for the fundamental result showing that the matrix orderings of $R$ with center $\wp$ are in one-to-one correspondence with the orderings of $K(\wp)$; there appears to be a large gap in Révész proof [R, Prop. 6 and Corollary]. (See Theorem 5 below.)

The fundamental idea is to think of how the Dieudonné determinant would behave if it were defined for rings. We shall see that for fields, the Dieudonné determinant induces a one-to-one correspondence between matrix orderings and ordinary orderings.

We write 1 and -1 for the matrices [1] and $[-1]$.

Definition. A matrix preordering of $R$ is a subset $T \subseteq \mathcal{M}(R)$ satisfying

(P1) $A, B \in T$ implies $A \nabla B \in T$ if it is defined.

(P2) $A, B \in T$ implies $A \dot{+} B \in T$.

(P3) $A+A \in T$ for all $A \in \mathcal{M}(R)$.

(P4) $-1 \notin T$.

(P5) $A+1 \in T$ implies $A \in T$.

(P6) $T$ contains all nonfull matrices.

Definition. A matrix ordering of $R$ is a subset $P \subseteq \mathcal{M}(R)$ satisfying

(O1) $A, B \in P$ implies $A \nabla B \in P$ if it is defined.

(O2) $A, B \in P$ implies $A \dot{+} B \in P$.

(O3) $A+A \in P$ for all $A \in \mathcal{M}(R)$.

(O4) $-1 \notin P$.

(O5) $P \cup-P=\mathcal{M}(R)$, where $-P=\{B \in \mathcal{M}(R) \mid \exists A \in P, A \nabla B$ nonfull $\}$.

(O6) $\wp=P \cap-P$ is a prime matrix ideal. It will be called the center of $P$.

It is not hard to show that the last two items in the definition of matrix ordering imply the last two items in the definition of matrix preordering. The set $-P$ can be defined in several different ways. The one above seems natural since the nonfull matrices fulfill a roll much like that of zero. In particular, a square matrix over a field is nonfull iff its Dieudonné determinant is zero. Two equivalent ways of writing $-P$ are included in our list of consequences of these definitions, both of which are much more useful in practice..

Other properties of matrix preorderings and orderings:

(1) For any matrix preordering $T$, we have $\{B \in \mathcal{M}(R) \mid \exists A \in T, A \nabla$ $B$ nonfull $\}=\left\{\left(-a \mid A^{\prime}\right) \mid\left(a \mid A^{\prime}\right) \in T\right\}$.

Proof. One containment is clear since $\left(0 \mid A^{\prime}\right)$ is nonfull. For the converse, we may assume $A=\left(a \mid A^{\prime}\right) \in T, B=\left(b \mid A^{\prime}\right)$ so that $\nabla$ is defined. As $\left(a+b \mid A^{\prime}\right)=A \nabla B$ is nonfull, (P6) implies that $A \nabla B \in T$, and that $\left(-a-b \mid A^{\prime}\right) \in T$. Since $\left(a \mid A^{\prime}\right) \in T$, (P1) implies $\left(-b \mid A^{\prime}\right)=\left(-a-b \mid A^{\prime}\right) \nabla\left(a \mid A^{\prime}\right) \in T$, whence $B$ lies in the right hand side as desired.

(2) For any matrix preordering $T$, we have $\{-1 \dot{+} A \mid A \in T\}$ equal to the two sets in (1). (This follows from the previous result and the fact that $1 \in T$.) This set will be denoted by $-T$.

(3) If $A$ is a nonfull matrix, then $A \in T \cap-T$ (by (P6)). 
(4) If $A \in T$, the result of adding any right multiple of one column (or left multiple of a row) to another column (row) again lies in $T$. (Use (P1), (P6) and follow [Co1, p. 397].)

(5) If $A \in T$, then interchanging two columns (rows) and changing the sign of one of them results in a matrix which again lies in $T$. (Use (P1), (P6) and follow [Co1, p. 397].)

(6) For $A, B \in \mathcal{M}(R)$ and $C$ of the appropriate size, $A \dot{+} B \in T$ iff $\left[\begin{array}{cc}A & 0 \\ C & B\end{array}\right] \in T$ (or equivalently, $\left[\begin{array}{cc}A & C \\ 0 & B\end{array}\right] \in T$ ). (Use (P2) and follow [Co1, p. 397-398].)

(7) If $A \in T$, the result of any even permutation of rows or columns again lies in $T$ by (5).

(8) If $A, B \in T$ of the same size, then $A B \in T$. More generally, $A B \in T \Longleftrightarrow$ $B A \in T$ for any $A, B \in \mathcal{M}(R)$ of the same size. (Same argument as [Co1, p. 398].)

(9) If $A \nabla B \in T \cap-T$ and $A \in T$, then $B \in-T$. (See proof of (1).)

(10) If $A \nabla B \in T \cap-T$, then $A+B \in-T$. (This follows from (9).)

(11) If $A \in T, B \in-T$, then $A \dot{+} B \in-T$.

One would like to be able to show that a maximal matrix preordering is a matrix ordering. This would allow a generalization of the Artin-Schreier criterion for existence of an ordering. This remains an open problem. One can easily handle matrix preorderings only with the prior assumption that $T \cap-T$ is a prime matrix ideal. This is essentially what Révész has done and what we do with matrix orderings below.

Let $D$ be a field. We shall asume that its characteristic is zero since we are only interested in ordered fields. Of special interest is the Dieudonné determinant (cf. [A, IV.1]), defined to be zero for singular square matrices, and defined modulo commutators otherwise. (Note that commutators are always squares, hence positive in any ordering!) Two properties of the Dieudonné determinant make it work for matrix orderings. The first is that $\operatorname{det}(A \nabla B) \subseteq \operatorname{det} A+\operatorname{det} B$ whenever $A \nabla B$ is defined [A, Theorem 4.5]. The second is the following proposition, in which $G L_{n} D$ is the general linear group of nonsingular $n \times n$ matrices and $D^{\times}$is the multiplicative group of nonzero elements of $D$.

Lemma 1. Let $n \geq 1$ be an integer. The group homomorphism $G L_{n} D \rightarrow D^{\times} / D^{\times 2}$ induced by the Dieudonné determinant is surjective with kernel equal to the subgroup $S_{n} D$ generated by all squares of $n \times n$ matrices.

Proof. We have the special linear group $S L_{n} D$ equal to the commutator subgroup $\left[G L_{n} D, G L_{n} D\right]$ by $\left[\mathbf{D}\right.$, p.138] and, in particular, contained in $S_{n} D$. Modulo $S L_{n} D$, each matrix has the form $\left[\begin{array}{cccc}d & & & \\ & 1 & & \\ & & \ddots & \\ & & & 1\end{array}\right]$ by $[\mathbf{A}$, Theorem 4.1]. Thus the 
mapping $d \mapsto\left[\begin{array}{cccc}d & & & \\ & 1 & & \\ & & \ddots & \\ & & & 1\end{array}\right]$ gives an inverse to $G L_{n} D / S_{n} D \rightarrow D^{\times} / D^{\times 2}$.

From these results, we obtain the characterization of matrix orderings for fields as an immediate corollary.

Theorem 2. Let $D$ be a field.

(a) Let $P_{0}$ be an ordering of $D$. Then $P_{0}$ extends to a matrix ordering $P$ of $D$ given by $A \in P \Longleftrightarrow \operatorname{det} A \subset P_{0}$.

(b) Any matrix ordering $P$ of $D$ induces an ordering $P_{0}$ defined by $a \in P_{0} \Longleftrightarrow$ $[a] \in P$.

(c) There is a one-to-one correspondence between orderings and matrix orderings of $D$.

Remark. In a similar manner, the ordinary determinant for commutative rings gives a one-to-one correspondence between orderings (in the sense of $[\mathbf{L}]$ ) and matrix orderings.

Rings and epic $R$-fields. Let $R$ be a ring and let $\wp$ be a prime matrix ideal of $R$. Our goal in this section is to establish the fundamental result that the matrix orderings of $R$ with center $\wp$ are in one-to-one correspondence with the orderings of the residue field $K(\wp)$. The proofs depend heavily on the construction of $K(\wp)$ detailed in [Co2, Chapter 4] and [Co1, Chapter 7]. Basically, this involves forming the localization $R_{\wp}$, a local ring with a homomorphism $R \rightarrow R_{\wp}$ with the property that every square matrix over $R$ not in $\wp$ becomes invertible over $R_{\wp}$. The elements of $R_{\wp}$ can be characterized by the fact that they are precisely the components of solutions of matrix equations $A u+a=0$ where $A$ lies in the image of $\mathcal{M}(R) \backslash \wp$, and $a$ is a column vector defined over the image of $R$ [Co1, Theorem 7.1.2; Co2, Theorem 4.2.1]. In fact, this can be done so that the element of $R_{\wp}$ is the first component of the solution and $a=e_{1}$, the first column of an identity matrix.

Now let $P$ be a matrix ordering of $R$ with center $\wp$. We define a subset $P_{D}$ of $D=K(\wp)$ as follows: for $x \in D$, choose $u \in R_{\wp}$ with image $x$ under the canonical mapping to the residue field; let $A \in \mathcal{M}(R) \backslash \wp$ be such that $A\left[\begin{array}{c}u \\ u_{2} \\ \vdots \\ u_{n}\end{array}\right]=r \in R^{n}$. Set $A_{1}=\left[\begin{array}{llll}r & a_{2} & \ldots & a_{n}\end{array}\right]$, where $a_{i}$ is the ith column of $A$. Define $x \in P_{D}$ if and only if $A \dot{+} A_{1} \in P$.

Theorem 3. $P_{D}$ is an ordering of $K(\wp)$.

Proof. We first show that the definition of $P_{D}$ is independent of the choice of $A$ and $u$. Consider two liftings of $x$, say $u, v$, with $A\left[\begin{array}{c}u \\ u^{\prime}\end{array}\right]=r$ and $B\left[\begin{array}{c}v \\ v^{\prime}\end{array}\right]=s$, where 
$u^{\prime}, v^{\prime}, r, s$ are column vectors. Set $M=\left[\begin{array}{c|cc}A & a_{1} & 0 \\ \hline 0 & B\end{array}\right]$, so that $M\left[\begin{array}{c}u-v \\ u^{\prime} \\ v \\ v^{\prime}\end{array}\right]=\left[\begin{array}{c}r \\ s\end{array}\right]$. With notation as above, $M_{1}=M\left[\begin{array}{cc}u-v & 0 \\ 0 & I\end{array}\right]\left[\begin{array}{cc}1 & 0 \\ u^{\prime} & I\end{array}\right]$, which becomes singular over $D$; thus $M_{1} \in \wp$. Now $M_{1}=N_{1} \nabla N_{2}$, where $N_{1}=\left[\begin{array}{c|cc}A_{1} & a_{1} & 0 \\ \hline 0 & B\end{array}\right]$ and $N_{2}=$ $\left[\begin{array}{c|c|cc}0 & a_{2} \ldots a_{n} & a_{1} & 0 \\ \hline s & 0 & B\end{array}\right]$. By (10) of the additional properties, we have $N_{1} \dot{+} N_{2} \in-P$. Switching columns 1 and $n+1$ of $N_{2}$ yields

$\left[\begin{array}{c|c|c|c}A_{1} & a_{1} & 0 & \multicolumn{2}{|c}{0} \\ \hline 0 & \multicolumn{2}{|c|}{B} & \multicolumn{2}{|c}{0} \\ \hline 0 & \frac{A}{b_{1}} & 0 & B_{1}\end{array}\right] \in P$.

Using (6) we obtain $A_{1} \dot{+} A \dot{+} B_{1} \dot{+} B \in P$, whence $A \dot{+} A_{1} \in P \Longleftrightarrow B \dot{+} B_{1} \in P$.

Next we check that $P_{D}$ is an ordering. The facts that $-1 \notin P_{D}$ and $P_{D} \cup-P_{D}=$ $D$ follow immediately from the corresponding facts for $P$. If $x \in P_{D} \cap-P_{D}$, then $A \dot{+} A_{1} \in P \cap-P$, which implies that $A_{1}$ is in $\wp$ since $A$ cannot be; i.e., $A_{1}$ becomes singular over $D$. Now, taking Dieudonné determinants, the equation

$$
A_{1}=A\left[\begin{array}{cc}
u & 0 \\
u^{\prime} & I
\end{array}\right]=A\left[\begin{array}{ll}
u & 0 \\
0 & I
\end{array}\right]\left[\begin{array}{cc}
1 & 0 \\
u^{\prime} & I
\end{array}\right]
$$

yields $x=0$. Finally, we check that $P_{D}$ is closed under multiplication and addition. Let $x, y \in P$ and lift them to $u, v \in R_{\wp}$ satisfying $A\left[\begin{array}{c}u \\ u^{\prime}\end{array}\right]=e_{1}$ and $B\left[\begin{array}{c}v \\ v^{\prime}\end{array}\right]=$ $e_{1}$, where $A \dot{+} A_{1}$ and $B \dot{+} B_{1}$ lie in $P$. Then $M\left[\begin{array}{c}u v \\ u^{\prime} v \\ v \\ v^{\prime}\end{array}\right]=\left[\begin{array}{c}0 \\ e_{1}\end{array}\right]$, where $M=$ $\left[\begin{array}{c|cc}A & -e_{1} & 0 \\ \hline 0 & B\end{array}\right]$. In this case $M_{1}=\left[\begin{array}{c|c|c|c}0 & a_{2} \ldots a_{n} & -e_{1} & 0 \\ \hline e_{1} & 0 & B\end{array}\right]$. Switching columns 1 and $n+1$ and changing the sign of the resulting first column shows that $M_{1} \in P$ iff $\left[\begin{array}{cc|c}A_{1} & 0 \\ \hline-b_{1} & 0 & B_{1}\end{array}\right] \in P$; i.e., iff $A_{1}+B_{1} \in P$. Also $M \in P \Longleftrightarrow A+B \in P$, so, since $A \dot{+} A_{1} \dot{+} B \dot{+} B_{1} \in P$, we have $M \dot{+} M_{1} \in P$ and hence $x y \in P_{D}$. Changing $M$ to $\left[\begin{array}{c|cc}A & -a_{1} & 0 \\ \hline 0 & B\end{array}\right]$, we obtain $M\left[\begin{array}{c}u+v \\ u^{\prime} \\ v \\ v^{\prime}\end{array}\right]=\left[\begin{array}{c}e_{1} \\ e_{1}\end{array}\right]$. In this case, $M_{1}=$ $\left[\begin{array}{c|cc}A_{1} & -a_{1} & 0 \\ \hline e_{1} & 0 & B\end{array}\right]$, which can be rewritten as $N_{1} \nabla N_{2}$, where $N_{1}=\left[\begin{array}{c|cc}A_{1} & -a_{1} & 0 \\ \hline 0 & B\end{array}\right]$ and $N_{2}=\left[\begin{array}{c|c|cc}0 & a_{2} \ldots a_{n} & -a_{1} & 0 \\ \hline e_{1} & 0 & B\end{array}\right]$. Clearly $N_{1} \in P \Longleftrightarrow A_{1} \dot{+} B \in P$. Also $N_{2} \in P \Longleftrightarrow A \dot{+} B_{1} \in P$, which can be seen by switching columns 1 and $n+1$ and changing a sign. Since $A \dot{+} A_{1}$ and $B \dot{+} B_{1}$ lie in $P$, we obtain $M \dot{+} N_{1}$ and $M \dot{+} N_{2}$ in $P$, so that $M \dot{+} M_{1}=M \dot{+}\left(N_{1} \nabla N_{2}\right)=\left(M \dot{+} N_{1}\right) \nabla\left(M \dot{+} N_{2}\right) \in P$.

Lifting orderings from $K(\wp)$ to $R$ is much simpler. 
Theorem 4. Let $D=K(\wp)$ be an epic $R$-field with matrix ordering $P_{D}$. Let $\phi: \mathcal{M}(R) \rightarrow \mathcal{M}(D)$ be the homomorphism induced by the canonical mapping from $R$ to the residue field $D$. Set $P \subset \mathcal{M}(R)$ equal to $\phi^{-1}\left(P_{D}\right)$. Then $P$ is a matrix ordering of $R$.

Proof. Let $A, B \in P$ with $A \nabla B$ defined. Then $\phi(A) \nabla \phi(B)$ is defined. Since $P_{D}$ is a matrix ordering, $\phi(A) \nabla \phi(B) \in P_{D}$, hence $A \nabla B \in P$. Conditions $(\mathrm{O} 2)$ and (O3) are verified similarly. Condition $(\mathrm{O} 4)$ is clear since $\phi([-1])=[-1]$. The final two conditions require the fact that $\phi^{-1}\left(-P_{D}\right)=-P$. To see this, one need only use the definition of minus given in $(2)$ and the fact that $\phi$ preserves $\dot{+}$. Condition (O6) then follows immediately, since $\wp=\left\{\phi^{-1}(A) \mid A\right.$ is singular $\}=$ $\phi^{-1}\left(P_{D} \cap-P_{D}\right)=\phi^{-1}\left(P_{D}\right) \cap \phi^{-1}\left(-P_{D}\right)=P \cap-P$. For condition (O5), let $A \in \mathcal{M}(R)$. Then $\phi(A) \in P_{D} \cup-P_{D}$, so that $A \in \phi^{-1}\left(P_{D} \cup-P_{D}\right)=P \cup-P$.

We now come to our main theorem, which shows that the definitions we have made really behave properly.

Theorem 5. The constructions of Theorems 3 and 4 induce a one-to-one correspondence between matrix orderings of a ring $R$ with center $\wp$ and orderings of the residue field $D=K(\wp)$.

Proof. We shall show that the given constructions are inverses of each other. We begin with an ordering $P_{0}$ of $D$; let $P_{0}$ also denote its unique extension to a matrix ordering as given by Theorem 2. Let $P$ be the matrix ordering of $R$ given by the construction of Theorem 4 and let $P_{D}$ be the ordering of $D$ induced by $P$ as in Theorem 3. We claim that $P_{0}=P_{D}$. Choose $0 \neq a \in P_{0}$. The element $a$ has a defining matrix equation $A\left[\begin{array}{c}u \\ u^{\prime}\end{array}\right]=e_{1}$, where $\bar{u}=a$. From the equation $A_{1}=$ $A\left[\begin{array}{ll}u & 0 \\ 0 & I\end{array}\right]\left[\begin{array}{cc}1 & 0 \\ u^{\prime} & I\end{array}\right]$, we obtain $a[D, D]=(\operatorname{det} A)^{-1} \operatorname{det} A_{1}=\operatorname{det}\left(A+A_{1}\right)(\operatorname{det} A)^{-2}$, which implies that $\operatorname{det}\left(A \dot{+} A_{1}\right) \subset P_{0}$ and thus that $A \dot{+} A_{1} \in P$. By definition, $a \in P_{D}$. Conversely, let $0 \neq a \in P_{D}$. Then, with the matrix notation above, $A \dot{+} A_{1} \in P$, hence $(\operatorname{det} A)\left(\operatorname{det} A_{1}\right) \subset P_{0}$. As above, $a[D, D]=(\operatorname{det} A)^{-1} \operatorname{det} A_{1}$, which is a subset of $P_{0}$, hence $a \in P_{0}$.

For the other composition of constructions, let $P$ be a matrix ordering of $R$ with $P \cap-P=\wp$. Let $P_{D}$ be the induced ordering of $D$ given by Theorem 3 ; we have seen that it extends uniquely to a matrix ordering of $D$ via the Dieudonné determinant. Let $P_{1}$ denote the inverse image of this matrix ordering, a matrix ordering of $R$. We know from the first part of the proof that $P_{1}$ also induces the ordering $P_{D}$ on the residue field. To see that $P_{1}$ is actually $P$, we proceed by induction on the number of rows $n$ of a matrix $A \in P$. We may assume that $A \notin \wp$. If $n=1$, we can write $A=[a], a \in R$. Using the equation $[a] \cdot[a]=\left[a^{2}\right]$, our defining condition for $P_{D}$ shows that, writing $A$ also for the image in $\mathcal{M}(D), \operatorname{det} A=\bar{a}$ is positive iff $\left[\begin{array}{cc}a & 0 \\ 0 & a^{2}\end{array}\right] \in P$; as $A=[a]$ and $\left[a^{2}\right]$ both lie in $P$, we obtain $\operatorname{det} A \in P_{D}$ and thus $A \in P_{1}$. Now assume that $n \geq 2$ is minimal such that $A \notin P_{1}$. Since $A$ becomes invertible over $R_{\wp}$, the equation $A\left[\begin{array}{c}u \\ u^{\prime}\end{array}\right]=e_{1}$ can be solved for $u \in R_{\wp}$ and $u^{\prime} \in R_{\wp}^{n-1}$. Now the sign of $\bar{u}$ (with respect to $P_{D}$ ) equals the sign of $A \dot{+} A_{1}$ 
with respect to both $P$ and $P_{1}$, where $A_{1}=\left[\begin{array}{cc}1 & * \\ 0 & A_{0}\end{array}\right]$ has the usual meaning, and $A_{0}$ is the $(n-1) \times(n-1)$ matrix formed by deleting the first row and column of $A$. Since $A$ has opposite signs with respect to $P$ and $P_{1}$, this must also be true of $A_{1}$. But $A_{1}$ and $A_{0}$ have the same sign with respect to any matrix ordering by $(6)$, whence $A_{0}$ must have opposite signs with respect to the two matrix orderings $P$ and $P_{1}$. As $A_{0}$ has only $n-1$ rows, this contradicts the induction hypothesis.

Corollary 6. Let $D$ be an epic $R$-field with singular kernel $\wp$. If two matrices in $\mathcal{M}(R)$ have images in $\mathcal{M}(D)$ with the same Dieudonné determinant (or even determinants which are congruent modulo sums of products of squares), then the matrices have the same sign with respect to every matrix ordering of $R$ with center $\wp$.

Remark. Let $T$ be a matrix preordering such that $\wp=T \cap-T$ is a prime matrix ideal. The construction and proof of Theorem 3, applied to $T$, show that $T$ induces a preordering on $K(\wp)$. This preordering is contained in an ordering of $K(\wp)$ which, by Theorem 4, pulls back to a matrix ordering of $R$ containing $T$.

The real spectrum. The set of orderings $X_{D}$ of a field $D$ has been studied by several authors, even in the noncommutative case (cf. $[\mathbf{C r}]$ ). As in the commutative case, this can be generalized to rings, namely to the set of all matrix orderings $X_{R}$ of a ring $R$. This set, together with the topology defined below, will be called the real spectrum of $R$. We assume that the set $X_{R}$ is nonempty.

The topology on $X_{R}$ is the one generated by taking, as a subbasis for the open sets, all sets of the form

$$
H(A)=\left\{P \in X_{R} \mid A \notin-P\right\}, \quad A \in \mathcal{M}(R)
$$

This will be called the Harrison topology .

Lemma 7. Let $T$ be a matrix preordering. Assume that $T$ satisfies

$$
A \dot{+} B \in-T \Longrightarrow A \in T \text { or } B \in T \text {. }
$$

Then $T$ is a matrix ordering.

Proof. Since $A \dot{+}(-1 \dot{+} A) \in-T$, we have either $A \in T$ or $A \in-T$; thus $T \cup-T=$ $\mathcal{M}(R)$. We must show that $\wp=T \cap-T$ is a prime matrix ideal. From the definition of matrix preordering, we know that $\wp$ contains the nonfull matrices, is closed under $\nabla$ and satisfies $A+1 \in \wp \Longrightarrow A \in \wp$. Now let $A \in \wp, B \in \mathcal{M}(R)$. We know that $B \in T \cup-T$; assume $B \in T$. Then $A \dot{+} B \in T$ and $A \dot{+} B \in-T$. Similarly if $B \in-T$. Therefore $\wp$ is a matrix ideal. To see that $\wp$ is prime, assume $A \dot{+} B \in \wp$ and $A \notin \wp$. We must show that $B \in \wp$. Without loss of generality, $A \notin T$. Since $A \dot{+} B \in-T$ and $A \dot{+}(-1 \dot{+} B) \in-T$, the hypothesis implies $B \in T$ and $-1 \dot{+} B \in T$, whence $B \in \wp$.

With the lemma above, we can give a proof that the real spectrum is compact (but not Hausdorff) which is very similar to the proof in the commutative case. 
Theorem 8. The real spectrum $X_{R}$ is compact.

Proof. Let $Y=\{0,1\}^{\mathcal{M}(R)}$, the set of all functions from $\mathcal{M}(R)$ to the discrete two point space $\{0,1\}$. We give $Y$ the product topology, so it is compact by Tychonoff's theorem. For any matrix ordering $P \in X_{R}$, we can define a function $f_{P}: \mathcal{M}(R) \rightarrow\{0,1\}$ as the characteristic function of $P \backslash(-P)$. The mapping $P \mapsto f_{P}$ is easily seen to give an embedding of $X_{R}$ into $Y$. The standard basis for the product topology on $Y$ consists of all sets of the form

$$
H_{\epsilon_{1}, \ldots, \epsilon_{n}}\left(A_{1}, \ldots, A_{n}\right)=\left\{f \in Y \mid(\forall i) f\left(A_{i}\right)=\epsilon_{i}\right\} \quad\left(A_{i} \in \mathcal{M}(R), \epsilon_{i} \in\{0,1\}\right)
$$

Note that $H_{1, \ldots, 1}\left(A_{1}, \ldots, A_{n}\right) \cap X_{R}=\bigcap H\left(A_{i}\right)$ is open in $X_{R}$. Thus if we can show that $X_{R}$ is closed in $Y$, we will know that it is compact with respect to the finer Tychonoff topology induced from $Y$ and hence is compact with respect to the Harrison topology as well. Let $f \in Y \backslash X_{R}$. We show that $X_{R}$ is closed by constructing a basic open set containing $f$ and missing $X_{R}$. Since $f \notin X_{R}$, the set $T=\{-1+A \mid f(A)=0\}$ must violate one of the six conditions in the definition of matrix preordering or the condition given in the previous lemma. Each of these leads immediately to the desired basic open set; e.g. for condition (P1), there exist matrices $A, B \in T$ with $A \nabla B$ defined, but not in $T$; thus $f \in$ $H_{0,0,1}(-1 \dot{+} A,-1 \dot{+} B,-1 \dot{+}(A \nabla B))$, an open set which contains no matrix ordering. Similarly, for (P6), there exists a nonfull matrix $A \notin T$, so $f \in H_{1}(-1+A)$; since $-1+A$ is again nonfull, $f_{P}(-1+A)$ must be zero for any matrix ordering $P$.

As in the commutative case, we have the immediate corollary (cf. [Cr]) that for any field $D$, the space of orderings $X_{D}$ is a Boolean space (compact, Hausdorff and totally disconnected) since the sets $H(A)$ and $H(-1 \dot{+} A)$ are complements, hence both closed and open.

\section{REFERENCES}

[A] E. Artin, Geometric algebra, Interscience Publishers, New York, 1957.

[Co1] P. M. Cohn, Free rings and their relations, Second edition, Academic Press, London, 1985.

[Co2] P. M. Cohn, Skew field constructions, London Math. Soc. Lecture Notes No. 27, Cambridge Univ. Press, New York, 1977.

[Co3] P. M. Cohn, Principles of non-commutative algebraic geometry, Rings and Geometry, R. Kaya, et al., eds., D. Reidel Publishing Co., Hingham, MA, 1985.

[Cr] T. Craven, Witt rings and orderings of skew fields, J. Algebra 77 (1982), 74-96.

[D] P. Draxl, Skew fields, London Math. Soc. Lecture Notes No. 81, Cambridge Univ. Press, New York, 1983.

[L] T. Y. Lam, An introduction to real algebra, Rocky Mountain J. Math. 14 (1984), 767-814.

[P] V. Powers, Higher level orders on noncommutative rings, J. Pure Appl. Algebra 67 (1990), $285-298$.

[R] G. Révész, Ordering epic R-fields, Manuscripta Math. 44 (1983), 109-130.

Department of Mathematics, University of Hawail, Honolulu, Hi 96822

E-mail address: tom@kahuna.math.hawaii.edu 